\title{
Nutraceuticals for Healthy Life
}

\section{Raman DANG}

Krupanidhi College of Pharmacy, \#12/1, Chikkabellandur, Carmelaram Post, Varthur Hobli, Bangalore, Karnataka, INDIA.

\begin{abstract}
The lifestyle has improved with economic development of the people. Side by side the major challenge are 'lifestyle diseases' that are due to food habits. Consumption of junk food has increased manifold, which has led to a number of diseases related to nutritional deficiencies. Hence they are shifting from synthetic ingredients towards organic foods and ingredients, which are obtained from natural sources. Of late, Nutraceuticals can play an important role in controlling them and also fulfill all the health requirements. They are incredible dietary supplements that help in prevention or treatment of disease, which is made from raw herbals. This is a rapidly growing industry with more than 100 million people using these natural products but as like drugs, there should be strict regulatory control for nutraceuticals. With extensive anecdotal data on exciting health results, nutraceutical promise significant contributions to disease prevention and allow humans to maintain an overall good health. Nearly two thirds of the world's 6.1 billion people rely on the healing power of plant based materials for many reasons-availability, affordability, safety or their belief in traditional cures. Hence the demand is irreversibly increasing. According to a new market research, global nutraceuticals market was valued at US\$ 165.62 billion in 2014 and is expected to reach US\$278.96 billion by 2021 , growing at a compound annual growth rate (CAGR) of $7.3 \%$ from 2015 to 2021. Indian markets growing at the rate of $21 \%$ per year and among that Amay, Dabur and Pfizer are well recognized. This indicates the people are accepting Nutraceuticals as medicine to make life healthy.
\end{abstract}

\section{INTRODUCTION}

The term "nutraceutical" combines two words

- "nutrient" (a nourishing food component) and "pharmaceutical" (a medical drug). The name was coined in 1989 by Stephen De Felice, founder and chairman of the Foundation for Innovation in Medicine, an American organization located in Cranford, New Jersey. A Nutraceutical is a pharmaceutical-grade and standardized nutrient. Nutraceuticals are regulated by FDA under the authority of the Federal Food, Drug, and Cosmetic Act.

\section{Categories of nutraceuticals}

The definition of nutraceuticals and related products generally depends on the source. They can be classified on the basis of their natural sources, pharmacological conditions, as well as chemical constitution of the products. Most often they are grouped in the following categories: dietary supplements, functional food, medicinal food, farmaceuticals.

A dietary supplement represents a product that contains nutrients derived from food products, and is often concentrated in liquid, capsule, powder or pill form. Although dietary supplements are regulated by the FDA as foods, their regulation differs from drugs and other foods.

According to their generally accepted definition, functional food is a category which includes whole foods and fortified, enriched or enhanced dietary components that may reduce the risk of chronic disease and provide a health-benefit beyond the traditional nutrients it contains.

Medical food is formulated to be consumed or administered internally, under the supervision of a qualified physician. Its intended use is a specific dietary management of a disease or condition for which distinctive
DOI: 10.5530/ijper.51.3s.2 Correspondence: Raman DANG, Krupanidhi College of Pharmacy, \#12/1, Chikkabellandur, Carmelaram Post, Varthur Hobli, Bangalore, Karnataka, INDIA.

Phone no: +919945846106 E-mail: dangraman2000@ gmail.com 
nutritional requirements are established by the medical evaluation (on the basis of recognized scientific principle).

Farmaceuticals are medically valuable components produced from modified agricultural crops or animals. The term is a combining of the words "farm" and "pharmaceuticals". Proponents of this concept are convinced that using crops (and possibly even animals) as pharmaceutical factories is much more cost effective than conventional methods, with higher revenue for agricultural producers.

\section{Potential health benefits}

Over the years nutraceuticals have attracted considerable interest due to their potential nutritional, safety and therapeutic effects. They could have a role in a plethora of biological processes, including antioxidant defenses, cell proliferation, gene expression, and safeguarding of mitochondrial integrity.

Therefore nutraceuticals may be used to improve health, prevent chronic diseases, postpone the aging process (and in turn increase life expectancy), or just support functions and integrity of the body. They are considered to be healthy sources for prevention of life threatening diseases such as diabetes, renal and gastrointestinal disorders, as well as different infections.

A wide range of nutraceuticals have been shown to impose crucial roles in immune status and susceptibility to certain disease states. They also exhibit diseases modifying indications related to oxidative stress including allergy, Alzheimer's disease, cardiovascular diseases, cancer, eye conditions, Parkinson's diseases and obesity.

\section{Healthy life style}

Nutricosmetics: Nutricosmetics are nutritional supplements which purpose to support the function and the structure of the skin. Many micronutrients have this effect. Vitamin C, for example, has a well established anti-oxidant effect that reduces the impact of free radicals in the skin.

Cosmeceuticals: It is the combination of cosmetics and pharmaceuticals. Cosmeceuticals are cosmetic products with bioactive ingredients purported to have medical or drug-like benefits. Dermatological research suggests that the bioactive ingredients used in cosmeceuticals have benefits beyond the traditional moisturizer

Nutrition: It is the science that interprets the interaction of nutrients and other substances in food in relation to maintenance, growth, reproduction, health and disease of an organism. It includes food intake, absorption, assimilation, biosynthesis, catabolism and excretion.

\section{Why?}

Our modern diets often lack the balance of nutrients our bodies require to maintain optimum health. Sugars and carbohydrates are at the top of the list of foods that have a negative impact on insulin sensitivity. Today, high levels of simple carbohydrates like bagels, pasta and pizza, as well as voluminous levels of sugar in found in oversized desserts, huge candy bars, etc. dominate the diets of many of us. We find "hidden sugar" in almost everything we eat from processed meats to sugar sprinkled into dried fruits.

Nutrition requires the complex interaction of many elements to be effective in fueling your body. Nutraceutical formulas help to fill the void by providing higher volumes, and the proper balance of vitamins, herbs, and minerals our systems need to maintain or restore health. For example, did you know that magnesium is required for more than 350 enzymatic reactions in the human body? Yet studies indicate that as few as one in four people have a dietary intake of magnesium that meets RDA levels.

Once upon a time, before the industrial revolution one could argue that our diets gave us what we needed. Higher quantities of fruits and vegetables were the rule, not the exception. Our carbohydrate intake took the form of whole grains that were produced in an environment that today would earn the label "organic." Natural fertilizers nurtured healthy, nutrient rich, tasty foods. That's dramatically changed.

Our Changing Earth Because of the increase in industrialized farming, our once nutrient-rich farmlands have been depleted through the widespread use of artificial fertilizers, irradiation of vegetables, over planting, and failure to practice healthy crop rotations. As a result, today's food supply is often lacking in the nutritional value our bodies expect and need. On the meat side of the equation we eat beef, pork, and poultry that have been raised on poor diets, drugs and hormones to speed production and increase weight and often, in inhumane environments.

This combination of lower quality food production and the increased popularity of an unbalanced, nutritionallydeficient diet has led to a need for additional nutritional support.

\section{Why complete and balanced nutrition is so important for you}

Let's look at a few examples of how nutritional balance is critical to diabetics.

Without adequate amounts of the mineral magnesium, the process of converting sugars from our diet into energy is impaired. Numerous studies have shown that 
people with uncorrected magnesium deficiency are much more likely to go on to develop insulin resistance and eventual diabetes within a relatively short period of time.

Chromium's primary job is to allow your cells to accept insulin. If you suffer from pre-diabetes, diabetes, or any other insulin-resistant disease, and if you are not ingesting enough chromium, your cells can't process insulin. Insulin and glucose levels become unbalanced and your health can deteriorate. When your cells receive the correct amount of chromium, they begin processing insulin more effectively and the complications of your disease may begin to reverse.

Alpha-Lipoic Acid is essential to breaking sugar down into a usable form that can be burned for energy. It also affects glucose transport, which results in increased insulin sensitivity and lower glucose levels. Lipoic acid is also an antioxidant with many vital protective functions, including extending the functional capacity of vitamin C, vitamin $\mathrm{E}$ and $\mathrm{CoQ} .{ }^{10}$ Lipoic acid is also one important way to raise glutathione levels, critical to people with diabetes that need to remove the toxic byproducts of excessive blood sugar.

Free Radicals are molecules that "react" quickly with proteins, fats and carbohydrates in the body. Our systems require free radicals, in excessive amounts these free radicals damage your cellular membrane, accelerate aging, and promote the disease process. Stress can play an important role in the creation of excess free radicals. The Insulite nutraceutical formulas include a combination of antioxidants, such as vitamin $\mathrm{C}$, alpha-lipoic acid, taurine, catechins, grape seed extract, quercitin, and others. These molecules have broad roles in protecting various cellular compartments against the production of free radicals that might impair energy production, damage cell membranes, or interfere with efficient cell repair.

\section{How Do Nutraceuticals Differ From Dietary Supplements?}

Nutraceuticals do more than just supplement the diet. They, as was pointed out, help with disease prevention and treatment. Theoretically, the appeal of nutraceuticals has to do with accomplishing treatment goals without side effects.

Using glucosamine as an example, by promoting joint health, it would seem by definition that it is more of a nutraceutical than dietary supplement. Are we essentially splitting hairs over terminology? Apparently so. According to Arthritis Research and Therapy, "the term nutraceutical has no regulatory definition and is not recognized by the U.S. Food and Drug Administration, which uses instead the term "dietary supplements." Some functional ingredients are sold as nutraceuticals in some countries but as drugs (that is, requiring medical prescription) in others."

The Merck Manual states, "The most commonly used alternative therapy is dietary supplements, which include medicinal herbs and nutraceuticals."

\section{Why consumers choose alternative over conventional remedies?

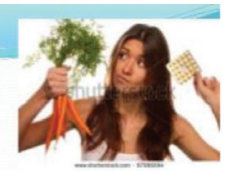 \\ Many patients are not satisfied with the treatment they are given by their doctors due to adverse effects or because it has been ineffective. \\ - Another reason for choosing alternative over conventional remedies is that patients may feel that conventional medicine is impersonal or technologically orientated. Some patients prefer to have personal control over their healthcare and therefore are happier to self-select than be told what to take by their doctor.}

\section{REGULATORY ASPECTS}

The primary set of rules governing the nutraceutical market is the Dietary Supplement Health and Education Act (DSHEA) passed in 1994. The Food Safety and Standard Rule, 2011 have been issued. Food Safety and Standard Authority has also issued regulations with respect to Licensing and registration of food business, manufacturing, packing and labeling, food product standard etc. The Food Safety and Standard Rule and Regulations are effective from August 2011. This act will encourage manufacturers for product Research and Development; develop reliable protocols and carryout clinical studies. Foreign Direct Investment Act passed recently in 2012 that also provide new opportunities for international firms to manufacture and sale nutraceutical products in India. Thus, there is only single authority to regulate production, distribution and marketing of nutraceuticals in India.

\section{CURRENT STATUS}

Nutraceutical food or food components that help in treatment and prevention of diseases are made from herbal/botanical raw material. This is rapidly growing industry (7-12\% per year) with more than millions of people in the world using these natural products. The 
global nutraceutical market to reach $\$ 450$ billion by 2015. According to recent analysis from Euro monitor, international global sales of health and wellness prod- ucts are on track to reach a record of about $\$ 1$ trillion by 2017, fueled by functional/ fortified products designed to offer specific health benefits.

\section{ABOUT AUTHOR}

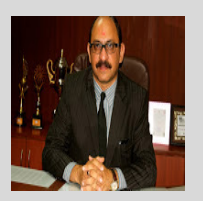

Prof. Dr. Raman Dang (M. Pharm, PhD, MBA), Principal, Krupanidhi College of Pharmacy, Bangalore, is a dynamic, hard-working, enthusiastic professional and responsible person in the field of Pharmaceutical Sciences. He has proven leadership abilities in working as a team, handling multiple tasks, great adaptability to any enterprise's environment.

$\mathrm{He}$ is having total 23 years of Teaching \& Research Experience. He is specialized in the area of cultivation and Standardization of Medicinal plants, Tissue culture of endangered plants, Pharmacological activities of plant extracts, Formulation and clinical investigation of plant products. In his career, he has guided $6 \mathrm{PhD}$ students, $32 \mathrm{M}$. Pharm students and co-guided 12 M. Pharm students. Not only academic, he proved his ability in Dabur industry, Ranbaxy where he worked as Research Associate for several months. He is active M. Pharm and Ph. D Examiner, Paper setter, Evaluator at various universities throughout India and appointed as chief and Deputy Chief for Post Graduate and Graduate University Examinations. Currently he holds the prestigious BOS Chairman post for PG section nominated by the Rajiv Gandhi University, Bangalore and is joint secretary for APTI (South zone).

Awards and acclaims: His carrier reflected with various prestigious awards and acclaims in which Gold Medal for Topping in Karnataka State for the Award of Diploma of Pharmacy, 1988, Best Paper Award at IPC, Indore. 1999, 1st runners up award at 61st FIP Conference held at Singapore, 2001 and Best Oral paper award at the international conference on botanicals held at Kolkata during 25th \& 26th Feb, 2005 are important. He has delivered lectures as invited speakers and chaired more than 12 scientific sessions for high profile scientific speakers in many national and international conferences. He was deputed as international scientific advisor for many conferences. Appointed chairperson for RAK-APTI International convention at Rasal AI Khaimah, UAE.

Paper presentation: He has presented more than 60 research papers in various national and international seminars, conferences and symposiums and organized workshops and conferences. He has appointed as International coordinator for GOFMAP that having 113 countries under its belt.

Publications: He has more than 60 research papers, 15 review papers, 3 conference proceedings in various peer reviewed national and international journals of repute. Apart from that he has more than 30 general articles contributed in various magazines especially in APTI bulletin. He has contributed 2 book chapters in edited book entitled "Recent progress in Medicinal Plant", Vol- 19, Published by Studium Press, LLC, USA, ISBN: 0-9656038-5-7".

Membership of bodies: He is associated with various prestigious bodies with life membership, among that membership in FIP, Society of Ethano pharmacology, APTI are highly appreciated. He serves as active member for academic council and faculty of pharmacy.

Editors and reviewers: $\mathrm{He}$ is serving as editorial board and reviewer committee member for more than 15 peer reviewed and national and international journals.

Special credentials: With his active efforts the college accreditated with quality certification by "The National Assessment and Accreditation Council (NAAC)" for maintaining sustained and uplifted quality education and actively involved in the finalizing of the MOU's between Teleradiology, Wipro and Green Chem. He serves as an expert committee and scrutiny member for various research projects under Rajiv Gandhi University of Health Sciences, Bangalore.

Cite this article: Dang R. Nutraceuticals for Healthy Life. Indian J of Pharmaceutical Education and Research. 2017;51(3)Suppl:S148-51. 